\title{
RECENT ADVANCES IN HYDROGELS FOR BIOMEDICAL APPLICATIONS
}

\author{
SUROJEET DAS ${ }^{1 *}$, VIVEK KUMAR ${ }^{1}$, RINI TIWARI ${ }^{1}$, LEENA SINGH $^{2}$, SACHIDANAND SINGH $^{1}$
}

${ }^{1}$ Faculty of Biotechnology, Institute of Bio-Sciences and Technology, Shri Ramswaroop Memorial University, Barabanki, Uttar Pradesh, India. ${ }^{2}$ Institute of Management, Commerce and Economics, Shri Ramswaroop Memorial University, Barabanki, Uttar Pradesh, India. Email: surojeetdas1990@gmail.com

Received: 14 June 2018, Revised and Accepted: 25 July 2018

ABSTRACT

Hydrogels are three-dimensional polymeric network, capable of entrapping substantial amounts of fluids. Hydrogels are formed due to physical or chemical cross-linking in different synthetic and natural polymers. Recently, hydrogels have been receiving much attention for biomedical applications due to their innate structure and compositional similarities to the extracellular matrix. Hydrogels fabricated from naturally derived materials provide an advantage for biomedical applications due to their innate cellular interactions and cellular-mediated biodegradation. Synthetic materials have the advantage of greater tunability when it comes to the properties of hydrogels. There has been considerable progress in recent years in addressing the clinical and pharmacological limitations of hydrogels for biomedical applications. The primary objective of this article is to review the classification of hydrogels based on their physical and chemical characteristics. It also reviews the technologies adopted for hydrogel fabrication and the different applications of hydrogels in the modern era.

Keywords: Hydrogels, Drug delivery, Tissue engineering, Soft materials, Controlled release, Cross-linked networky.

(C) 2018 The Authors. Published by Innovare Academic Sciences Pvt Ltd. This is an open access article under the CC BY license (http://creativecommons. org/licenses/by/4. 0/) DOI: http://dx.doi.org/10.22159/ajpcr.2018.v11i11.27921

\section{INTRODUCTION}

Hydrogel (also called as aquagel) is a network of polymer chains, threedimensional water swollen structures. They are composed mainly by hydrophilic homopolymers or copolymers. They swell but are insoluble in water, sometimes found as a colloid in which water is the dispersal medium. Insolubility is due to chemical or physical cross-links which also provide shape. Hydrogels are super permeable (they can contain over $99 \%$ water) and closely conjure up as natural living tissue due to their significant water content, absorbance, and soft texture [1-3].

Since the pioneering work of Wichterle and Lim, 1960, on cross-linked 2-hydroxyethyl methacrylate (HEMA) hydrogels [4], biomaterial scientists have shown deep research interest in hydrogels. In the last few years, the smart properties of hydrogel coupled with a high versatility and a high tunability of material's properties lead to innate research and management of hydrogels. Polymer hydrogel scaffolds have proved to be one of the most efficient matrixes in tissue engineering for repairing and regenerating a wide variety of tissues or organs. Hydrogels have been used in different biomedical disciplines for over 50 years. Hydrogel applications range from basic usage as lubricants, contact lenses, and burn healing agents to higher performances such as controlled drug delivery systems (DDSs) and specialized carriers for various macromolecules [5,6].

In late years, extensive efforts have been devoted to fabricating hydrogels as novel DDS, since it is a convenient means of sitespecific and time-controlled delivery of therapeutic agents $[7,8]$. Hydrogels have gained considerable interest as drug containers or discharge rate controlling barriers [9-14]. Recently developed biosensitive hydrogels perform one of the most essential functions of drug delivery and biomedical applications, where the kickback to external stimuli drives the release of the drugs [1]. New (smart) hydrogel systems with various chemically and structurally responsive components demonstrate sensitivity to external stimuli including temperature, $\mathrm{pH}$, ionic concentration, light, magnetic fields, electrical fields, and chemicals. Two or more stimuli-responsive procedures have been combined to develop polymers with multiple responsive properties. Smart polymer hydrogels change their structural and volume phase transition as a response to external stimuli resulting in an enormous potential for scientific observations and for various advanced technological applications. An innovative approach with the production of $\mathrm{pH}$-sensitive hydrogel helped for controlling release rates as well as for directing to a site of absorption or action.

Hydrogels have also proved to be an attractive recognition element for intelligent DDS, targeted DDS, and microfluidic devices. Bioadhesive gels assisted in localizing protein/peptide release systems through oral and pulmonary routes, and they consequently grab immense sphere for drug targeting and prolonging the drug action at a targeted spot. The review is directed toward different structural parameters that impact hydrogel fabrication and toward a discussion of the latest developments in current research being pushed over hydrogels [1].

\section{TYPES OF HYDROGELS}

Hydrogels have been subcategorized based on natural and synthetic polymers used for fabrication as shown in Fig. 1.

\section{Natural hydrogels}

Collagen, fibrin, gelatin, etc., are the natural polymers used to fabricate hydrogels. Natural polymers offer diverse advantageous properties such as inherent biocompatibility, biodegradability, and biologically recognizable moieties that support cellular activities. However, these polymers fail to provide sufficient mechanical properties and may contain pathogens or evoke immune/inflammatory response $[15,16]$.

\section{Synthetic hydrogels}

Polymers such as polyvinyl alcohol (PVA), polyethylene oxide (PEO), and polyacrylic acid (PAA) are examples of synthetic materials used for hydrogel fabrication. Précised structures that can be modified to yield tailorable degradability and functionality are the exclusive features of synthetic polymers. By altering the chemical composition and fabrication techniques, the structure of hydrogels can be manipulated. Nevertheless, synthetic hydrogels do not possess inherent bioactive properties as that of natural polymers $[3,16]$. 


\section{DIFFERENT METHODS FOR HYDROGEL FABRICATION}

\section{Physical methods}

Gelation induced through hydrogen bonding, gelation brought by sonication, hydrogel formation from polymer solution through ionic interactions, heating or cooling of polymer solution, and freeze thawing of polymer solution are the various physical methods applicable for fabrication of hydrogel. Table 1 depicts the various physical methods to synthesize hydrogels.

\section{Chemical methods}

The formation of hydrogels through chemical method involves crosslinking of natural and synthetic polymers. This is achieved through the reaction of their functional groups such as $\mathrm{OH}, \mathrm{COOH}$, and $\mathrm{NH} 2$ where aldehyde acts as cross-linker (e.g., glutaraldehyde and adipic acid dihydrazide). Grafting of monomers on the backbone of the polymers or the use of a cross-linking agent to link two polymer chains is the elemental chemical cross-linking methods [17]. Table 1 includes different chemical methods for hydrogel synthesis.

\section{Cross-linking using high-energy irradiation}

Gamma rays as high-energy radiations for polymerizing unsaturated compounds electron beams have been widely used. Employing irradiation, the conversion of water-soluble polymers can be derivatized with vinyl groups to construct hydrogels [18]. A mixture of a monofunctional acrylate like acryloyl-l-proline methyl ester in the presence of suitable cross-linker can be used to fabricate hydrogels by radiation-induced polymerization. Further, cross-linking of various polymers such as PVA, poly(ethylene glycol) (PEG), and poly(acrylic acid) can be achieved by high-energy irradiation [19]. The properties of so-formed gels, such as their swelling and permeability, greatly lean on the radiation dose and the concentration of polymer. Free radical polymerization of acrylic acid on the backbone of carboxymethyl cellulose (CMC) was used to construct hydrogel of CMC. This was done by grafting CMC with acrylic acid in the existence of electron beam irradiation, in aqueous solution [20].

\section{Enzymatic methods}

Cross-linking through enzymatic methods emerged as another acceptable method for the construction of hydrogels which helped to overcome the limitations raised by physical methods. Hydrogel fabrication techniques like cross-linking by radiation-induced cytotoxicity in the so-formed gels, whereas cross-linking by physical methods produced gels with low mechanical strength and stability.
Hence, these limitations induced path for cross-linking by naturally occurring enzymes [21].

\section{Transglutaminase}

Transglutaminase offers a mild alternative to chemical cross-linking. Transglutaminase relates to the family of thiol enzymes which induce isopeptide bond formation [22]. Gelatin-based hydrogels formed using transglutaminase can be employed for incorporation of cells depicting good cytocompatibility and attractive features for tissue engineering applications [23,24].

\section{Tyrosinase}

Tyrosinase-catalyzed hydrogels are mechanically weak and have been observed to be formed in the presence of chitosan only. According to studies, tyrosinase possesses a feature of fast degradation which makes it suitable to be used as glue, wound dressings, and also in protein immobilization $[25,26]$.

\section{Phosphopantetheinyl transferase}

The use of phosphopantetheinyl transferase for catalyzing hydrogel formation from polymers was recently reported [27]. The cross-linking catalyzed by phosphopantetheinyl transferase possesses the potential to integrate bioactive peptides, specifically the receptor-binding motifs for integrin, like RGDs (Arg-Gly-Asp), enabling cell attachment [28].

\section{PROPERTIES OF HYDROGELS}

\section{Swelling properties}

All polymer chains in hydrogels are deliberated as one molecule when cross-linked to each other either physically or chemically regardless of its size. The physical texture of the hydrogel can be diversified with a slight variation in environmental parameters such as $\mathrm{pH}$, temperature, electric signal, and presence of enzymes or other ionic species. Hydrogel response fluctuates with the external environmental $\mathrm{pH}$ when acidic or basic functional groups are cross-linked under the fabrication routine. Thus, functional groups dictate swelling profile and degree of ionization leading to the shift in volume [29].

Thermoresponsive behavior of the poly(N-isopropylacrylamide) (PNIPAM) hydrogel was affected by copolymerization of $\mathrm{N}$-isopropylacrylamide (NIPAM) with a hydrophilic monomer $\mathrm{N}$, $\mathrm{N}$-dimethylacrylamide (DMAM) and a hydrophobic monomer methyl
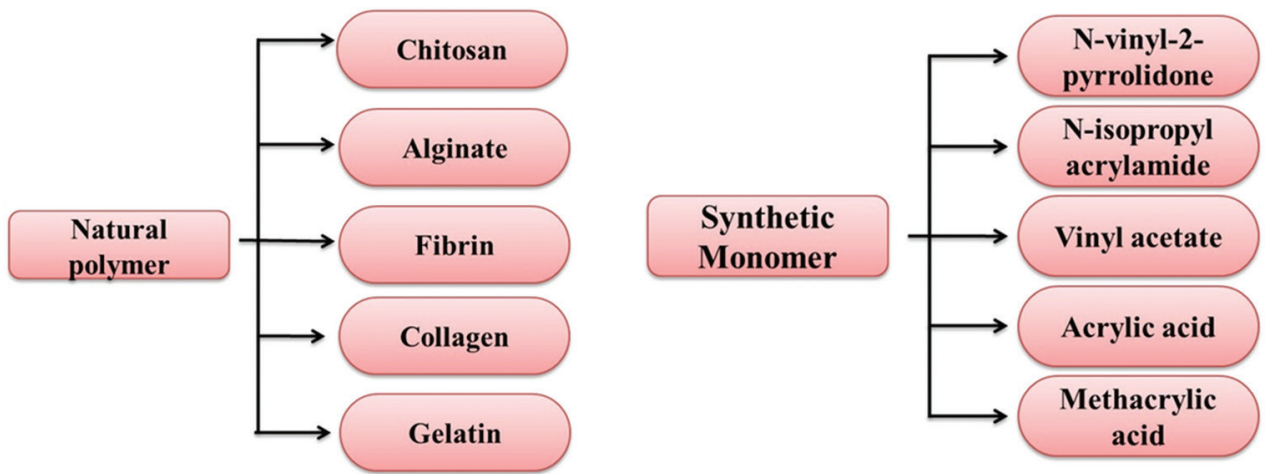

Fig. 1: Distinct types of hydrogels based on polymer type

Table 1: Methods for synthesizing physically and chemically cross-linked hydrogels [17]

Physically cross-linked hydrogels

Ionic interactions (alginate)

Hydrophobic interactions (poly (ethylene oxide)-poly (propylene oxide), poly (ethylene oxide)

Hydrogen-bonding interactions (poly (acrylic acid)

Stereocomplexation (enantiomeric lactic acid)

Supramolecular chemistry (inclusion complex)
Chemically cross-linked hydrogels

Polymerization (acryl group)

Radiation (gamma rays)

Small molecule cross-linking (glutaraldehyde)

Polymer-polymer cross-linking (condensation) Chemically cross-linked hydrogels 
methacrylate (MMA). NIPAM-DMAM copolymer hydrogel with higher lower critical solution temperature (LCST) is more open with waterswollen behavior above their LCST, and the NIPAM-MMA copolymer hydrogels with lower LCST are less open along with water shrunken nature below their LCST when compared with PNIPAM homopolymer hydrogel [30].

\section{Mechanical properties}

The construction of hydrogels should be such that it fulfills the mechanical property and retains its physical texture during the delivery of therapeutic moieties for the predestined course of time. The mechanical properties which are desired in a hydrogel can be achieved by altering the degree of cross-linking. The estimation of cross-linking density of hydrogels was made possible on the basis of Young modulus and Flory's theory [31]; consequently, the generalized Maxwell model helped to control hydrogel linear viscoelastic range along with the relaxation spectra [29].

\section{Biocompatible properties}

Hydrogels need to be biocompatible and non-toxic in nature for effective biomedical applications. Biocompatibility of hydrogels dwells in two basic aspects. First, biosafety includes the exemption of cytotoxicity, mutagenesis, and carcinogenesis, and second, biofunctionality, i.e., the capability of material to execute the distinct task for which it is intended. The presence of synthetic cross-linkers and initiators used in the polymerizations of naturally derived monomers and prepolymers are subjugated to the same toxicity concerns as purely synthetic hydrogels; therefore, natural polymers are frequently regarded to have superior biocompatibility over synthetic ones [29].

\section{APPLICATIONS OF HYDROGELS}

\section{Drug delivery}

Hydrogels have played an efficient role as DDS since they possess advantageous properties such as excellent tissue compatibility, easy manipulation, and solute permeability [32]. Since decades, hydrogels have been utilized for the fabrication of DDS. In the aqueous medium, the drug enclosed within the hydrogel dissolves as soon as the water penetrates into the system. Drug within the hydrogel diffuses out in the surrounding aqueous medium by means of diffusion [33]. Smart polymeric hydrogels deliver drugs at the right time and accurate concentration in response to external stimulus [34].

Active molecules of a drug contain a broad spectrum of proteins and peptides, which are released through hydrogel carriers as they bind less strongly with the drugs. The release of drugs in the body at distinct sites and times would reduce unwanted side effects, eventually improving the medical treatment [35].

Smart hydrogels (Fig. 2) change their properties when in contact with distinct stimuli for controlling drug delivery. Drug release profiles under temperature responsive hydrogel display a stepwise temperature change, thus being extremely studied to obtain an "on-off" profile [35]. At the molecular level, the physical and chemical properties such as permeability, enviro-responsive nature, surface functionality, biodegradability, and surface biorecognition sites were optimized by the researchers for controlled drug delivery applications [7]. Drug incorporation, release of model molecules, and release kinetics are the factors which are taken into consideration while fabricating a hydrogel for drug delivery.

\section{Drug incorporation}

Ligands which are metabolically cleavable and have specific affinity for active agents are used to perform drug loading by physical entrapment, with either post-fabrication drug absorption or sometimes in situ encapsulation. Materials employed as hydrogel carriers for osteoinductive factors include alginate, gelatin, hyaluronic acid (HA), and synthetic or natural PEG-based polymers. Drug loading just before cross-linking helps to maintain its stability as well as its biological potency. Thus, during the fabrication process, this prevents the drug from exposure to rough fabrication conditions or from leaching out [36].

\section{Release of model molecules}

Biomolecule release occurs by the process of diffusion of molecules acting as a function of porosity, degradation, or swelling of hydrogels. Physical cross-linking through ultraviolet radiation or thermal and ionic or covalent cross-linking (by acrylate or thiol chemistry, glutaraldehyde or carbodiimide, etc.) methods can be employed to estimate the permeability and the swelling of hydrogel for release of model molecule [37]. Cytocompatibility and also the effective control over small-sized biomolecules and proteins are decreased by enhanced cross-linking of polymers. Chemically-controlled delivery of growth factors is the most efficient system for providing reproducible release profiles from hydrogels, where it usually gets affected by mode and degradation rate of hydrogel, interactions between hydrogel and molecule, and also by the inclusion of varied phases (including polymers or even particulate carriers) or of cleavable sequences in the hydrogel network [38]. Hydrogels bearing cross-linkable labile ester bonds can be produced by copolymerizing PEG with chemical

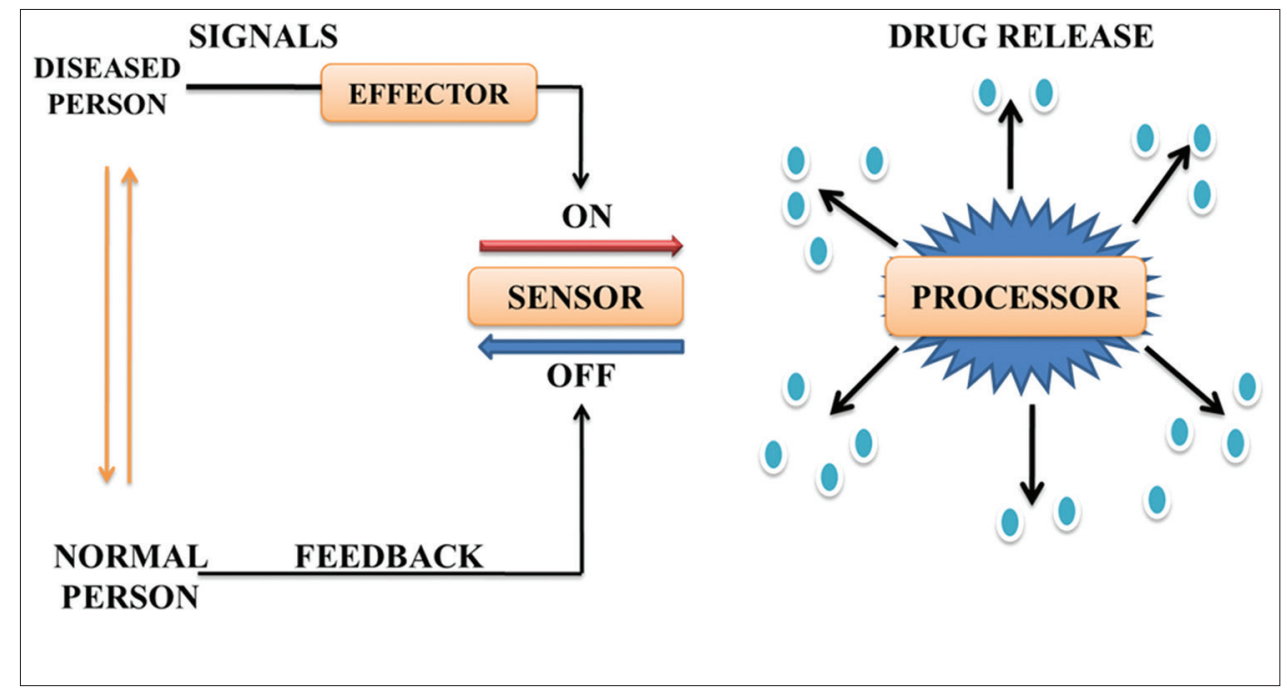

Fig. 2: Design concept for "Smart" drug delivery systems with sensor, processor, and effector functions 
intermediaries which have either end-capped acrylate or the sebacic acid acrylate group or either by PLA or poly(gamma-glutamic acid) (PGA). Lyophilizing silk hydrogel loaded with antibody was employed to produce sustained delivery matrix called lyogels focusing over the interactions occurring between antibodies and its feasibility as a potent delivery system for the therapeutic protein [36].

\section{Kinetics of drug release from hydrogels}

In DDS, drugs can be encased or immersed within a hydrogel corresponding to several diverse types of controlled release systems. Further, controlled release systems were subcategorized into diffusioncontrolled systems and swelling-controlled systems [39].

Diffusion-controlled delivery systems are subdivided into reservoir devices and matrix devices. Diffusion is the key phenomena for release of the drugs in both the cases. A reservoir delivery system usually consists of capsules, cylinders, spheres, or slabs where drug core is enclosed within a hydrogel membrane. The drug concentration difference must remain constant for the sake of uniform release rate.

In matrix systems, the drug is fully dispersed all through the threedimensional structure of the hydrogel. Constituents required for the construction of matrix tablets include a compression of a mixture of drug and polymer powders. Considering the release profiles, macromolecular mesh or water-filled pores are responsible for drug release throughout the matrix. The release pace is proportional to the square root of time in preference to the constant time-independent rate available with reservoir systems [40]

The drug is dispersed within a glassy polymer as a matrix device in swelling-controlled release systems. The polymer begins to swell as it comes in unity with water or another biofluid. The drug can now diffuse out of the swollen rubbery area of the polymer as the glass transition temperature of the polymer is lowered allowing a relaxation of molecular chains [41]. In some cases, anomalous transport occurs which is the combination of swelling-controlled release as well as diffusion [39].

\section{Wound healing and wound dressing}

Hydrogels have the ability to absorb and retain foreign bodies such as bacteria in its network structure. Thus, it can act as a moist wound dressing material. In further applications, hydrogels have the tendency to adsorb and eliminate dead tissues which prove to be a novel feature for defending wounds. It also helps in enhancing fibroblast proliferation by reducing fluid loss from the wound surface - [33]. Hydrogels lack mechanical strength but fulfill all the criteria to be used as an outstanding wound dressing material. Permeability, flexibility, and occlusive properties gradually depend on the swelling characteristics of various engineered hydrogels. The first hydrogel-based wound dressing material formulated was hydron [42-45]. Some other examples of dressings prepared from HEMA monomers in combination with other polymers such as PEG, acrylamide, or agar are Gel perm, Intrasite, Biolex, and Vigilon $[46,47]$.

\section{Tissue engineering}

Hydrogels likely resemble natural tissues thus emerged as one of the abundantly used scaffold biomaterials [35]. Tissue engineering has received increased focus in recent decades due to the use of resorbable hydrogels. This is since the properties of a hydrogel can be modified effortlessly. Tissue engineering involves both synthetic and natural polymer hydrogels to restore cartilage, tendon, ligament, skin, blood vessels, and heart valves [48]. Naturally derived hydrogels comprise collagen, silk, keratin, agarose, fibrin, alginate, chitosan, gelatin, and HA, whereas the synthetic hydrogels include polyurethanes, PEO, PVA, PAAc, and poly (propylene fumarate-co-ethylene glycol) $[35,49,50]$.

Hydrogels also act as an immune isolation barrier for microencapsulation technology [51] along with acting as a scaffold for tissue engineering [52]. Microencapsulation involves a process where allogeneic or xenogeneic cells are harbored from the host's immune system using a semipermeable membrane. In hydrogels, polymeric microcapsules containing cells are enclosed in such a way to intensify the functionality of transplanted constructs [51]. Hydrogels act as support structures for cell growth and function and deliver signals to cells $[53,54]$.

To construct injectable thermoresponsive hydrogels, thermoresponsive polymers are mixed at room temperature with the cells, thus optimized to be used as substrates that facilitate cell growth and proliferation and then be injected into the body. The polymer forms a physical gel as the temperature increases (to $37^{\circ} \mathrm{C}$ ) which is quite above the polymer's LCST on injection. The cells are encapsulated within the three-dimensional structure of the gel [34]. Three-dimensional corneal implant, tracheal gland cells, etc., are examples of collagen-coated tissue culture inserts. Type III collagen mixed with fibronectin or with matrigel is frequently used for culturing normal mature liver cells which also involve fibrillar collagen-coated scaffolds (e.g., filter, swatch of nylon, transwell, and biodegradable microcarrier) [55].

Biocompatible/biodegradable materials along with other hydrogels were found to expand in contact with liquid when coated over nails, screws, pins, hip and knee replacements, etc., in the case of orthopedic fasteners and replacements. In some approaches, replacements made up of stainless steel, metal alloys, titanium, or cobalt chromium, coated along hydrogels proved to be a vital step in improving metal-polymer adhesion. Natural or synthetic polymers are raised as constructs for replacement of cartilage, where hydrogel phase often serves as carrier for cells and therapeutics. Hydrogels resemble cartilage tissues as they possess high water content that ranges between $75 \%$ and $80 \%$ by weight [56]. This helps in rapid diffusion of soluble nutrients and supports to retain rounded cell morphologies [57,58]. Injectable solutions are widely accepted as constructs which are delivered by invasive process $[59,60]$. PEG-based hydrogel/calcium phosphate nanocomposite developed by Schlichting ., 2011 [60], emerged as useful temporary reinforcement to fill cracks in cartilage occurred by trauma [61]. To mechanically stabilize the severely injured cartilage and prevent further damage to the cartilage (which occurs during the following 1-2 weeks after the injury), the photopolymerizing copolymer solution was introduced under this area [61].

\section{Gene delivery}

Gene therapy is the delivery of desired therapeutic genes into cells aiming repair, replacement, and regulation of defective genes to avoid diseases [34]. Poly-L-lysine, PEG, polyamidoamine dendrimer, polyethylenimine, PGA, poly(lactic acid) (PLA), and poly(di-lactic acidco-glycolic acid) (PLGA) are examples of polymers used by scientists for gene delivery [61]. Researchers have used PEG-PLGA-PEG hydrogel for delivery of plasmid-beta 1 gene in diabetic mouse model that enhanced wound healing [62]. Transfection is a process of gene delivery by polymeric carriers that involve first, complexation of DNA and polymer, addition of DNA/polymer complex into cells for a period. Elimination of end complexes from the cells and last, incubation time where cells are kept incubating for a period till the results are recorded.

\section{Cancer therapy}

Hydrogel microparticles have the ability to extend and target drugs, which reduce the body's burden remarkably [63]. Since faster and greater swelling is needed, superporous hydrogels will be more useful than normal hydrogels. For direct administration, such as in the case of intratumoral administration, hydrogels can be directly transplanted or injected into the tumor. This increases the efficacy of the anticancer agent. Hydrogel implantation can be used as an alternative approach for multiple dose conventional therapies [1].

Thermoresponsive hydrogels have been reported as delivery systems for chemotherapeutic agents because they can be injected as a liquid into the tumor and then gelled at body temperature. OncoGel ${ }^{\mathrm{TM}}$ is an example of an anticancer drug-loaded thermoresponsive hydrogel 
which is intended for the localized delivery of paclitaxel into the tumor minimizing significantly the side effects associated with conventional administration of the drug. The benefits of OncoGel ${ }^{\mathrm{TM}}$ include direct deposition of the formulation in the targeted site using a syringe in case of superficially accessible tumors or more specialized tools for deeper solid tumors. [64,65]. Magnetic hydrogel nanocomposites (NCs) are a new emerging class of stimuli-responsive hydrogels being developed for in vivo drug release for tumor therapy. Incorporation of magnetic nanoparticles such as iron oxide $\left(\mathrm{Fe}_{3} \mathrm{O}_{4}\right)$ in a hydrogel matrix results in a tunable NC that can be distantly controlled by a magnetic field [66].

\section{Diagnostics}

Hydrogels serve as carriers with engineered release properties, as coatings with targeting capabilities, as coatings with stealth properties or combinations, and as whole, therefore, researchers introduced hydrogel systems in imaging applications [7]. For example, intracellular imaging pertains a novel carrier for quantum dots based on nanogels [67]. In addition, radioisotopes for radiation delivery devices were in capsulated by hydrogel coatings $[68,69]$.

\section{Microarray technology}

A variety of applications that enclose hydrophilic polymers requires control of surface properties and spatial location. Hydrogels along with hydrophilic polymers have been designed for controlling cell-cell interactions, altering surfaces, immobilizing cells, and protein within hydrogel microstructures for usage in microarray. Thus, to immobilize cells in particular regions of a substrate, photocrosslinkable PEG hydrogels have been used. This task can be performed by patterning PEG on a substrate $[70,72]$. Collagen gels have also been molded to pattern cells [73]. Polysaccharides being one of the natural hydrophilic polymers were broadly used as patterning materials. As per one of the studies, hydrophilic polysaccharides such as HA possess the tendency to form adsorbed monolayers on surfaces of hydrophilic substrates [74,75].

\section{Medical and biological sensors}

Hydrogels can be incorporated with biosensors where the constructed gel expands or contracts in response to actions delivered by the specified substance or condition. Ultrasensitive bio-microelectromechanical systems (MEMSs) sensor platform was designed and tailored over silicon microcantilevers with the help of environmentally responsive hydrogel $[76,77]$. Microscale MEMS sensor device was the first, where actuation was controlled by an intelligent polymer network [7]. A hydrogelbased photonic crystal was designed by Holtz and Asher as glucose sensor that sensed glucose in patients suffering from diabetes mellitus [78]. Table 2 depicts the recent applications of hydrogels for biosensors.

\section{Ophthalmology}

Most of the ophthalmic drugs on an average constitute eye drops or eye ointments. These drugs face certain drawbacks and, hence, fail to deliver drugs efficaciously to the intraocular tissue [79]. On the other hand, corneal tissue irritation is one of the familiar problems raised by eye ointments [80]. Therefore, exploring alternate drug delivery strategy helps to overcome these drawbacks by exploring particles, films, hydrogels, and contact lenses [81].

Hydrogels being the principal material for soft contact lenses emerged as one of the most potent drug carriers for ophthalmic drugs because

Table 2: Recent developments in hydrogel-based biosensors [76-78]

\begin{tabular}{llll}
\hline $\begin{array}{l}\text { Stimulus } \\
\text { Glucose }\end{array}$ & $\begin{array}{l}\text { Hydrogel } \\
\text { PA-PEG }\end{array}$ & $\begin{array}{l}\text { Application } \\
\text { Glucose } \\
\text { biosensor } \\
\text { Pntibiotin } \\
\text { biosensor } \\
\text { Live cell } \\
\text { biosensor }\end{array}$ & $\begin{array}{l}\text { Output signal } \\
\text { Optical, color }\end{array}$ \\
Peptide & PEG & $\begin{array}{l}\text { Optical, focusing } \\
\text { fluorescence }\end{array}$ \\
\hline
\end{tabular}

PEG: Poly (ethylene glycol) of their high water content [82,83]. Hydrogel lenses are dipped into drug solution for effective drug loading $[84,85]$. Conventional HEMA-based hydrogel lenses have been diagnosed for ophthalmic drug $[82,86]$. Under one of the approaches, HEMA-based hydrogels copolymerized with methacrylic acid were diagnosed for the release of timolol, a therapeutic drug in the treatment of glaucoma. Bausch and Lomb, in 1999, introduced the first silicone hydrogel lens in the market by the name PureVision. Silicon-based SCLs were developed to increase oxygen permeability. Since 1970, silicon elastomers pertaining high oxygen permeability have been in trend as a key material for contact lenses. Various other experimental approaches under contact lenses were investigated to raise the loading efficiency that embraces embedding drug-loaded nanoparticles, microemulsions, or liposomes in the hydrogel lens [87-90].

\section{Neural therapy}

In human physiology, the central nervous system (CNS) possesses limited intrinsic regenerative capacity. Hence, various therapies fail to provide significant functional recovery in diseases and injuries of the CNS including those in the brain, spinal cord, and retina. Diffusion of molecules into the brain by traditional oral or intravenous routes has always been one of the challenges, as delivery of these therapies to the CNS is interrupted by blood-brain barriers. Injectable hydrogels thus provide a nominal invasive, localized, and void-filling platform for drug delivery to the CNS [91].

In other popular approach, liposomes and lipid microtubules [92,93] or polymeric microspheres [94,95] were combined with hydrogel for drug delivery methods. Drug delivery to CNS in context to hydrophobic liposomes or microspheres yielded extended release times for hydrophilic molecules [96]. Extracellular matrix of connective, epithelial, and neural tissues is highly loaded with HA, thus making it widely used material for tissue regeneration. HA interacts with cells principally through CD44 and receptor for HAmediated motility surface receptors $[97,98]$ and also plays key roles in various metabolic and cellular processes such as cell proliferation, morphogenesis, inflammation, and wound repair. PNIPAAm is also being commonly used as a temperature responsive DDS $[99,100]$ and PLA as micro/nanoparticles DDSs are the widely studied hydrogels by the researchers [101].

\section{Immunoisolation}

Immunoisolation simply deals with encapsulating a living cell within a semipermeable barrier that permits the bidirectional passage of gases and metabolites but regulating the entry of large molecules and immunoglobulin. Immunoisolation mechanically chunks the entry of immunocytes which help to swamp the limitations of implanting free cells [102]. Based on the basis of immune isolation and immune protection, Chang, 1964, was the first to construct a bioartificial organ using hydrogel. Immunoisolation of transplanted cells secreting hormones, neurotransmitters, growth factor, and other bioactive cellular secretary products has raised the advancement of various gel devices for wider purposes [103].

\section{CONCLUSION}

Recently, many hydrogel-based networks have been designed and tailored to meet the needs for different biomedical applications. Considerable progress has been made in improving the properties of hydrogels used for drug delivery, expanding the range of drugs and kinetics which can be achieved using hydrogel-based delivery vehicles. However, numerous challenges remain to enhance the clinical applicability of hydrogels as drug carriers. There is a need for continued improvement in delivery of more sensitive molecules such as proteins, antibodies, or nucleic acid which can easily unfold itself by the interaction with the hydrogel delivery vehicle. Hydrogel delivery systems can leverage therapeutically beneficial outcomes of drug delivery and have found clinical use. Hydrogel can provide spatial and temporal control over the release of various therapeutic agents including small molecule drugs, macromolecules drugs, and cells. 
Due to the tunable physical properties, controllable degradability and capability to protect labile drugs from degradation, hydrogel serves as a platform on which various encapsulated drugs occur to control drug release. Self-assembled hydrogel and hydrogels for tumor targeting and imaging are to be explored at a greater pace. The valuable addition of efficient DDS in comparison to the newly found drug can benefit economically as well as drastically reduce the duration of time taken to develop the new drug.

\section{AUTHORS' CONTRIBUTION}

All the authors contributed equally in the conceptualization and execution of the review article.

\section{CONFLICTS OF INTEREST}

The authors declared that they have no conflicts of interest.

\section{REFERENCES}

1. Kashyap N, Kumar N, Kumar MR. Hydrogels for pharmaceutical and biomedical applications. Crit Rev Ther Drug Carrier Syst 2005;22:107- 49.

2. Dill KA. Strengthening biomedicine's roots. Nature 1999;400:30910.

3. Peppas NA, Bures P, Leobandung W, Ichikawa H. Hydrogels in pharmaceutical formulations. Eur J Pharm Biopharm 2000;50:27-46.

4. Wichterle 0, Lim D. Hydrophilic gels for biological use. Nature 1960;185:117-8.

5. Dumitriu S. Polymeric Biomaterials Revised and Expanded. 2nd ed. New York, Basel: Marcel Dekker; 2002.

6. Iwona G, Helena J. Review: Synthetic polymer hydrogels for biomedical applications. Chem Chem Tech 2010:4:297-304.

7. Peppas NA, Zach HJ, Khademhosseini A, Langer R. Hydrogels in biology and medicine: From molecular principles to bio nanotechnology. Adv Mater 2006;18:1345-60.

8. Huang X, Brazel CS. On the importance and mechanisms of burst release in matrix-controlled drug delivery systems. J Control Rel 2001;73:121- 136

9. Hoffman AS. Hydrogels for biomedical applications. Adv Drug Deliv Rev 2002;54:3-12.

10. Hoare TR, Kohane DS. Hydrogels in drug delivery: Progress and challenges. Polymer 2008;49:1993-2007.

11. Baroli B. Hydrogels for tissue engineering and delivery of tissueinducing substances. J Pharm Sci 2007;96:2197-223.

12. Ganta S, Devalapally H, Shahiwala A, Amiji M. A review of stimuliresponsive nanocarriers for drug and gene delivery. J Control Release 2008;126:187-204

13. Schmaljohann D. Thermo- and pH-responsive polymers in drug delivery. Adv Drug Deliv Rev 2006;58:1655-70.

14. Peppas NA, Khare AR. Preparation, structure and diffusional behavior of hydrogels in controlled release. Adv Drug Deliv Rev 1993;11:1-35.

15. Davis KA, Anseth KS. Controlled release from crosslinked degradable networks. Crit Rev Ther Drug Carrier Syst 2002;19:385-423.

16. Lin CC, Metters AT. Hydrogels in controlled release formulations: Network design and mathematical modeling. Adv Drug Deliv Rev 2006;58:1379- 408

17. Chauhan S, Harikumar SL, Kanupriya. Hydrogels: A smart drug delivery system. IJRPC 2012; 2(3):603-614.

18. Nguyen KT, West JL. Photopolymerizable hydrogels for tissue engineering applications. Biomaterials 2002;23:4307-14.

19. Carenza M, Caliceti P, Veronese FM, Martellini F, Higac OZ, Yoshida M, et al. Poly (acryloyl-L-proline methyl ester) hydrogels obtained by radiation polymerization for the controlled release of drugs. Rad Phys Chem 2000;57:471-5.

20. Said HM, Alla SG, El-Naggar AW. Synthesis and characterization of novel gels based on carboxymethyl cellulose/acrylic acid prepared by electron beam irradiation. React Funct Polym 2004;61:397-404.

21. Teixeira LS, Feijen J, van Blitterswijk CA, Dijkstra PJ, Karperien M. Enzyme-catalyzed crosslinkable hydrogels: Emerging strategies for tissue engineering. Biomaterials 2012;33:1281-90.

22. Pisano JJ, Finlayson JS, Peyton MP. Cross-link in fibrin polymerized by factor 13: Epsilon-(gamma-glutamyl)lysine. Science 1968;160:892-3.

23. Yung CW, Bentley WE, Barbari TA. Diffusion of interleukin-2 from cells overlaid with cytocompatible enzyme-crosslinked gelatin hydrogels. J Biomed Mater Res A 2010;95:25-32.

24. Yung CW, Wu LQ, Tullman JA, Payne GF, Bentley WE, Barbari TA, Transglutaminase crosslinked gelatin as a tissue engineering scaffold. J Biomed Mater Res A 2007;83:1039-46.

25. Yamada K, Chen T, Kumar G, Vesnovsky O, Topoleski LD, Payne GF, et al. Chitosan based water-resistant adhesive. Analogy to mussel glue. Biomacromolecules 2000;1:252-8.

26. Demolliens A, Boucher C, Durocher Y, Jolicoeur M, Buschmann MD, De Crescenzo G, . Tyrosinase-catalyzed synthesis of a universal coilchitosan bioconjugate for protein immobilization. Bioconjug Chem 2008;19:1849-54.

27. Mosiewicz KA, Johnsson K, Lutolf MP. Phosphopantetheinyl transferase-catalyzed formation of bioactive hydrogels for tissue engineering. J Am Chem Soc 2010;132:5972-4.

28. D’Souza SE, Ginsberg MH, Plow EF. Arginyl-glycyl-aspartic acid (RGD): A cell adhesion motif. Trends Biochem Sci 1991;16:246-50.

29. Das N. Preparation methods and properties of hydrogel: A review. Int J Pharm Pharm Sci 2013;5:112-7.

30. Iwai K, Hanasaki K, Yamamoto M. Fluorescence label studies of thermo-responsive poly ( $\mathrm{N}$-isopropylacrylamide) hydrogels. J Lumin 2000;1289:87-9.

31. Grassi M, Sandolo C, Perin D, Coviello T, Lapasin R, Grassi G, et al. Structural characterization of calcium alginate matrices by means of mechanical and release tests. Molecules 2009;14:3003-17.

32. Paleos GA. What are Hydrogels? Pennsylvania: Pittsburgh Plastics Manufacturing; 2012

33. Pal K, Banthia AK, Majumdar DK. Polymeric hydrogels: Characterization and biomedical applications - A mini review. Des Monomers Polym 2009;12:197-220.

34. Ward MA, Georgiou TK. Thermo responsive polymers for biomedical applications. Polymers 2011;3:1215-42.

35. Ebara M, Kotsuchibashi Y, Narain R, Idota N, Kim YJ, Hoffman JM, et al. Smart Biomaterials. $1^{\text {st }}$ ed. Berlin: Springer NIMS Monographs; 2014. p. 1-7.

36. Vo TN, Kasper FK, Mikos AG. Strategies for controlled delivery of growth factors and cells for bone regeneration. Adv Drug Deliv Rev 2012;64:1292-309.

37. Peppas NA. Hydrogels in Medicine and Pharmacy. Boca Raton: CRC Press; 1986. p. 180

38. Taylor SJ, McDonald JW, Sakiyama-Elbert SE. Controlled release of neurotrophin-3 from fibrin gels for spinal cord injury. J Control Release 2008;98:281-94.

39. Lowman AM, Peppas NA. Hydrogels. Encyclopedia Controlled Drug Deliv 1999;1:397-418.

40. Bierbrauer F. Hydrogel Drug Delivery: Diffusion Models. University of Wollongong. Internal Report 2005. Available from: https:// www.e-space.mmu.ac.uk/615529/. [Last retrieved on 2018 May $10]$.

41. Lowman AM. Smart Pharmaceuticals. Available from: http://wwwgateway.vpr.drexel.edu/files/NewEh/htmls/lowman.pdf. [Last retrieved on 2018 May 10].

42. Silva AK, Richard C, Bessodes M, Scherman D, Merten OW. Growth factor delivery approaches in hydrogels. Biomacromolecules 2009;10:9-18

43. Ratnaparkhi PK, Prajapati VK, Jani GK, Solanki HK. Recent expansions in an emergent novel drug delivery technology: Hydrogel. World J Pharm Pharm Sci 2015;4:678-701.

44. Kishida A, Ikada Y. Hydrogels for biomedical and pharmaceutical applications. In: Dumitriu S, editor. Polymeric Biomaterials. $2^{\text {nd }}$ ed. Ch. 6. New York: Marcel Dekker; 2002.

45. Brown AS. Hydron for burns. Plast Reconstruct Surg 1981;67:8101.

46. Yates DW, Hadfield JM. Clinical experience with a new hydrogel wound dressing. Injury 1984;16:23-4.

47. Myers JA. Delperm: A nontextile wound dressing. Pharm J 1983;230:263.

48. Lee KY, Mooney DJ. Hydrogels for tissue engineering. Chem Rev 2001;101:1869-79.

49. Kumaran P, Gupta A, Sharma S. Synthesis of wound-healing keratin hydrogels using chicken feathers proteins and its properties. Int J Pharm Pharm Sci 2017;9:171-8.

50. Azevedo EP. Chitosan hydrogels for drug delivery and tissue engineering applications. Int J Pharm Pharm Sci 2015;7:8-14.

51. Lim F, Sun AM. Microencapsulated islets as bioartificial endocrine pancreas. Science 1980;210:908-10. 
52. Hasan A, Waters R, Roula B, Dana R, Yara S, Alexandre T, et al. Engineered biomaterials to enhance stem cell-based cardiac tissue engineering and therapy. Macromol Biosci 2016;16:958-77.

53. Elisseeff J, McIntosh W, Fu K, Blunk BT, Langer R. Controlledrelease of IGF-I and TGF-beta1 in a photopolymerizing hydrogel for cartilage tissue engineering. J Orthop Res 2001;19:1098-104.

54. Anseth KS, Burdick JA. New directions in photopolymerizable biomaterials. MRS Bull 2002;27:130-6.

55. Zhang R, Ma PX. Processing of polymer scaffolds: Phase separation. In: Atala A, Lanza RP, editors. Methods of Tissue Engineering. $1^{\text {st }}$ ed. San Diego, California: Academia Press; 2006. p. 715.

56. Darling EM, Athanasiou KA. Biomechanical strategies for articular cartilage regeneration. Ann Biomed Eng 2003;31:1114-24.

57. Baksh D, Yao R, Tuan RS. Comparison of proliferative and multilineage differentiation potential of human mesenchymal stem cells derived from umbilical cord and bone marrow. Stem Cells 2007;25:1384-92.

58. Coleman RM, Case ND, Guldberg RE. Hydrogel effects on bone marrow stromal cell response to chondrogenic growth factors. Biomaterials 2007;28:2077-86

59. Tan H, Marra KG. Injectable biodegradable hydrogels for tissue engineering applications. Materials 2010;3:1746-67.

60. Schlichting KE, Copeland-Johnson TM, Goodman M, Lipert RJ, Prozorov T, Liu X, . Synthesis of a novel photopolymerized nanocomposite hydrogel for treatment of acute mechanical damage to cartilage. Acta Biomater 2011;7:3094-100.

61. Choi JS, Park JS. Design elements of polymeric gene carrier in biomaterials for delivery and targeting of proteins and nucleic acids. In: Mahato RI, editor. 2nd ed. Boca Raton: CRC Press; 2005. p. 643-62.

62. Lee PY, Li Z, Huang L. Thermosensitive hydrogel as a tgf-beta1 gene delivery vehicle enhances diabetic wound healing. Pharm Res 2003;20:1995-2000.

63. Teder H, Johnsson CJ. The effect of different dosage of degradable starch microsphere (spherex) on the distribution of doxorubicin regionally administered to the rat. Anticancer Res 1993;13:2161-4.

64. Lapidus RG, Dang W, Rosen DM, Gady AM, Zabelinka Y, O’Meally $\mathrm{R}$, . Anti-tumor effect of combination therapy with intratumoral controlled-release paclitaxel (PACLIMER microspheres) and radiation. Prostate 2004;58:291-8.

65. Safran H, Akerman P, Cioffi W, Gaissert H, Joseph P, King T, Paclitaxel and concurrent radiation therapy for locally advanced adenocarcinomas of the pancreas, stomach, and gastroesophageal junction. Semin Radiat Oncol 1999;9:53-7.

66. Mawad D, Boughton EA, Boughton P, Lauto A. Advances in hydrogels applied to degenerative diseases. Curr Pharm Des 2012;18:2558-75.

67. Kuang M, Wang D, Bao H, Gao M, Mohwald H, Jiang M. Fabrication of multicolor-encoded microspheres by tagging semiconductor nanocrystals to hydrogel spheres. Adv Mat 2005;17:267-70.

68. Azhdarinia A, Yang DJ, Yu DF, Mendez R, Oh C, Kohanim S, Regional radiochemotherapy using in situ hydrogel. Pharm Res 2005;22:776-83.

69. Qu X, Weinberger J. Deposition of (90)YPO(4) and (144)CePO(4) radioisotopes on polymer surfaces for radiation delivery devices. J Biomed Mater Res 2002;63:98-105.

70. Koh WG, Revzin A, Simonian A, Reeves T, Pishko M. Control of mammalian cell and bacteria adhesion substrates micro patterned on PEG hydrogels. Biomed Microdevices 2003;5:9-11.

71. Jon SY, Seong JH, Khademhosseini A, Tran TN, Laibinis PE, Langer R. Construction of nonbiofouling surfaces by polymeric selfassembled monolayers. Langmuir 2003;19:9989-93.

72. Khademhosseini S, Jon SY, Tran TN, Eng G, Yeh J, Seong J, Langer R. Direct patterening of protein and cell resistant polymeric monolayers and microstructures. Adv Mater 2004;15:1995-2000.

73. Tang MD, Golden AP, Tien J. Molding of three-dimensional microstructures of gels. J Am Chem Soc 2003;125:12988-9.

74. Suh KY, Khademhosseini A, Yang JM, Eng G, Langer R. Soft lithographic patterning of hyaluronic acid on hydrophilic substrates using molding and printing. Adv Mat 2004;16:584-8.

75. Khademhosseini A, Suh KY, Yang JM, Eng G, Yeh J, Levenberg S, Layer-by-layer deposition of hyaluronic acid and poly-L-lysine for patterned cell co-cultures. Biomaterials 2004;25:3583-92.

76. Bashir R, Hilt JZ, Elibol O, Gupta A, Peppas NA. Micromechanical cantilever as an ultrasensitive $\mathrm{pH}$ microsensor. App Phy Let
2002;81:3091-92.

77. Hilt JZ, Gupta A, Bashir R, Peppas NA. Ultrasensitive biomems sensors based on microcantilevers patterned with environmentally responsive hydrogels. Biomed Microdevices 2003;5:177-84.

78. Holtz JH, Asher SA. Polymerized colloidal crystal hydrogel films as intelligent chemical sensing materials. Nature 1997;389:829-32.

79. Gupta S, Samanta MK, Raichur AM. Dual-drug delivery system based on in situ gel-forming nanosuspension of forskolin to enhance antiglaucoma efficacy. AAPS PharmSciTech 2010;11:322-35.

80. Burstein NL. Corneal cytotoxicity of topically applied drugs, vehicles and preservatives. Surv Ophthalmol 1980;25:15-30.

81. Gaudana R, Jwala J, Boddu SH, Mitra AK. Recent perspectives in ocular drug delivery. Pharm Res 2009;26:1197-216.

82. Xinming L, Yingde C, Lloyd AW, Mikhalovsky SV, Sandeman SR, Howel CA,et al. Polymeric hydrogels for novel contact lens-based ophthalmic drug delivery systems: A review. Cont Lens Anterior Eye 2008;31:57- 64 .

83. Tranoudis I, Efron N. Water properties of soft contact lens materials. Cont Lens Anterior Eye 2004;27:193-208.

84. Hu X, Hao L, Wang H, Yang X, Zhang G, Wang G, et al. Hydrogel contact lens for extended delivery of ophthalmic drugs. Int J Polymer Sci 2011;2011:1-9.

85. Singh K, Nair AB, Kumar A, Kumria R. Novel approaches in formulation and drug delivery using contact lenses. J B Clin Pharm 2011;2:87-101.

86. Li C, Chauhan A. Ocular transport model for ophthalmic delivery of timolol through p-HEMA contact lenses. J Drug Deliv Sci Technol 2007;17:69- 79.

87. Gulsen D, Chauhan A. Dispersion of microemulsion drops in HEMA hydrogel: A potential ophthalmic drug delivery vehicle. Int J Pharm 2005;292:95-117.

88. Gulsen D, Chauhan A. Ophthalmic drug delivery through contact lenses. Invest Ophthalmol Vis Sci 2004;45:2342-7.

89. Sharif Makhmalzadeh B, Salimi A, Niroomand A. Loratadine-loaded thermoresponsive hydrogel: Characterization and ex-vivo rabbit cornea permeability studies. Iran J Pharm Res 2018;17:460-9.

90. Sri B, Ashok V, Arkendu C. As a review on hydrogels as drug delivery in the pharmaceutical field. Int J Pharm Chem Sci 2012;1:642-61.

91. Pakulska MM, Ballios BG, Shoichet MS. Injectable hydrogels for central nervous system therapy. Biomed Mater 2012;7:024101.

92. Maherani B, Arab-Tehrany E, Mozafari MR, Gaiani C, Linder M. Liposomes: A review of manufacturing techniques and targeting strategies. Curr Nanosci 2011;7:436-52.

93. Lee $\mathrm{H}$, McKeon RJ, Bellamkonda RV. Sustained delivery of thermostabilized chABC enhances axonal sprouting and functional recovery after spinal cord injury. Proc Natl Acad Sci U S A 2010;107:3340-5.

94. Lampe KJ, Kern DS, Mahoney MJ, Bjugstad KB. The administration of BDNF and GDNF to the brain via PLGA microparticles patterned within a degradable PEG-based hydrogel: Protein distribution and the glial response J Biomed Mater Res A 2011;96:595-607.

95. Baumann MD, Kang CE, Tator CH, Shoichet MS. Intrathecal delivery of a polymeric nanocomposite hydrogel after spinal cord injury. Biomaterials 2010;31:7631-9.

96. Ali A, Ahmed S. Recent advances in edible polymer based hydrogels as a sustainable alternative to conventional polymers. J Agric Food Chem 2018;66:6940-67.

97. Johl SS, Burgett RA. Dermal filler agents: A practical review. Curr Opin Ophthalmol 2006;17:471-9.

98. Sahoo S, Chung C, Khetan S, Burdick JA. Hydrolytically degradable hyaluronic acid hydrogels with controlled temporal structures. Biomacromolecules 2008;9:1088-92.

99. Gil ES, Hudson SM. Stimuli-responsive polymers and their bioconjugates. Prog Polym Sci 2004;29:1173-222.

100. Rzaev ZM, Dincer S, Piskin E. Functional copolymers of $\mathrm{N}$-isopropylacrylamide for bioengineering applications. Prog Polym Sci 2007;32:534-95

101. Piantino J, Burdick JA, Goldberg D, Langer R, Benowitz LI. An injectable, biodegradable hydrogel for trophic factor delivery enhances axonal rewiring and improves performance after spinal cord injury. Exp Neurol 2006;201:359-67.

102. Ríhová B. Immunocompatibility and biocompatibility of cell delivery systems. Adv Drug Deliv Rev 2000;42:65-80.

103. Chang TM. Semipermeable microcapsules. Science 1964;146:524-5. 